\title{
ERROR ANALYSIS OF DISPLACEMENT GRADIENTS VIA FINITE ELEMENT APPROXIMATION OF DIGITAL IMAGE CORRELATION SYSTEM
}

\author{
Shahriyar Beizaee*, Kaspar J. Willam*, Giovanna Xotta ${ }^{\dagger}$ and Reza Mousavi* \\ ${ }^{*}$ University of Houston \\ Houston, TX USA \\ e-mail: sbeizaee@uh.edu,kwillam@uh.edu,m.reza.mousavi@gmail.com \\ †Universita of Padova \\ Padova, Italy \\ e-mail: giovanna.xotta@dicea.unipd.it
}

Key words: Digital Image Correlation, Least Square Approximation, Finite Element Mapping

\begin{abstract}
In the field of experimental solid mechanics, conventional strain measurement devices such as LVDT and strain gauges provide mean values of strains and displacements at selected locations and gauge lengths; this result is inadequate for the evaluation of a non homogeneous material behaviour. Consequently, during the last decades various full-field non-contact measurement techniques have been proposed for the material characterization and have become more and more popular in the experimental mechanics community. In this work, the Digital Image Correlation (DIC) non-interferometric technique has been used to monitor experiments on aluminum flat bars and to measure displacement distribution on the surface of the specimen for further evaluation and calculation of strains.

The results obtained by a Digital Image Correlation System are assessed and the error associated with the post processing of the experimental field data, obtained through the use of the Aramis software, is evaluated and analyzed with the aid of a least square approximation code. This code uses a finite element approximation of the displacement field in order to cover all the target points. A least square approximation of these data is performed and the best nodal displacement values are determined. Based on the nodal data, infinitesimal and finite strain distributions are determined over the surface image window of the specimen. It is observed that this post processing technique provides better results near perforations and edges that are not sensitive to the density of the captured displacement data.
\end{abstract}

\section{INTRODUCTION}

Digital Image Correlation system is used in the scientific community for a wide variety of applications. Chu et al. [1] explained the theory of deformation and digital correlation to form a measurement technique and used interpolation techniques to extend the range of measurements. Lava et al. [2] analyzed the errors from different implementation of digital image cor- relation by imposing FE displacement fields on an undeformed image. Yang et al. [3] monitored the decrease in crack spacing with the increase in FRP reinforcement ratio in reinforced concrete by using digital image correlation system. Corr et al. [4] used DIC as a fracture mechanics tool to examine the bond between CFRP and concrete. Pan et al. [5] reviewed the methodologies of the 2D DIC technique for displacement 
and strain estimation. Ghiassi et al. [6] used DIC to investigate the strains and deformation for uniaxial test and shear debonding of FRP and Masonry. Images from DIC can be also used into expert systems to investigate damage causes. Champiri et al. [7, 8] developed an expert system for concrete structures. Roncella et al. [9] conducted a comparative analysis between cross-correlation and least square matching by performing a test on a brick mounted on a micrometric sledge.

This paper proposes an alternative methodology to post process the displacenent data acquired by a photogrammetric non-contact digital image correlation system. This is a lateral outcome of several studies on cementitious and metallic material where digital image correlation was used to capture strain and displacement distributions [10-16]. During these studies, it was observed that the post processing software of the DIC system is very sensitive to facet size near perforations and edges. In order to resolve this problem, a method is introduced which is based on finite element analysis and least square method. This method uses the extracted displacement data and maps it on a finite element mesh using a least square approximation. This paper is divided into three parts. The first part explains the experiments performed on metallic specimen. The second part explains the methodology that processes the raw data obtained by DIC and the last part compares the results obtained by the new technique with the strains obtained from the Aramis post processing.

\section{Experiments on Metallic Flat Bars}

This section provides an overview of the experiments that were performed on metallic flat bars and explains the process of data acquisition using a Digital Image Correlation (DIC) system. Several perforated and unperforated flat bar specimens made of mild steel and aluminum were prepared based on ASTM B557 [17], ASTM A370 [18] and ASTM E8 [19] standards. The geometrical dimensions of these flat bars are presented in Figure 1. Each mate- rial had five different sets of coupons, one nonperforated and four perforated coupons. The circular perforations range from $1 / 16$ to $1 / 4$ of an inch. Each test configuration consists of four flat bars. Two flat bars were tested under uniaxial tension for each case, if the results were not consistent and/or different from existing information, the third and fourth coupons were tested.

The Tinius Olsen and Shore Western testing machines were used to perform uniaxial tensile experiments on these coupons. DIC system was used to measure the displacement and strain distributions on the surface of these specimens. These uniaxial experiments were displacement controlled and the rate of the deformation was kept constant in order to eliminate possible strain rate effects. The test setup is shown in Figure 1. The flat bars were placed inside the grips and the photogrammetric noncontact digital image correlation system was mounted in front of the specimen according to the instructions of the GOM optical measuring techniques company [20].



Figure 1: Dimension of the flat bars and test setup

The specimens were painted in white and then speckles of black paint was sprayed on the specimen. The speckle pattern of a painted mild steel specimen is depicted in Figure 2. Two cameras were placed in front of the specimen in order to have a 3D measurement field. These cameras observe the deformation of the specimen through the images captured at time inter- 
vals by various rectangular image details known as facets. These facets are quadrilaterals that include a portion of the specimen in form of pixels that cover $n$ by $n$ pixels of the specimen which are equally distanced from one another. The 2D coordinates of the facets are calculated based on the corners points of the quadrilateral and center of the facet is calculated by averaging the four corners. Using the information from left and right cameras, the 3D coordinates of the facet points are calculated.

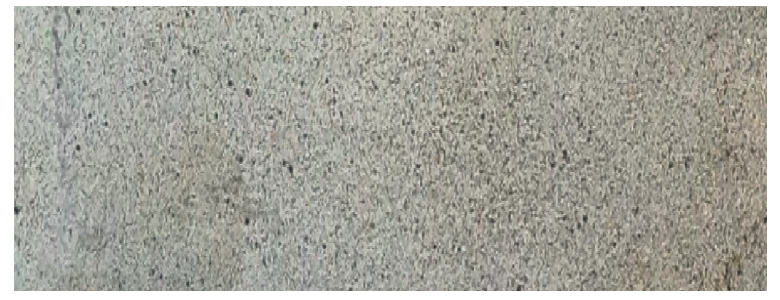

Figure 2: Speckle pattern on the specimen, white background with black dots

In order to examine the accuracy of the displacement data obtained by the DIC system, linear variable differential transformer (LVDT) was used in conjunction with the DIC. A series of uniaxial tensile experiments were performed using both LVDT and DIC. The comparison of the displacement data obtained by these instruments is presented in Figure 3. Test 1 represents the sample without perforation and tests 2 to 5 are for the perforated samples.

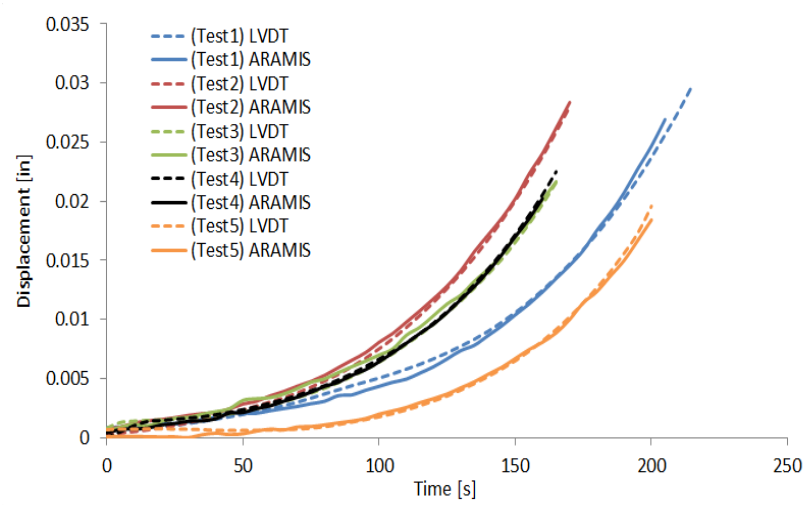

Figure 3: Comparison of the deformation data obtained by DIC and LVDT

Root mean square deviation between the global displacement data obtained by using DIC and LVDT measurements demonstrates a very good agreement. Therefore the displacement data obtained by DIC are considered valid and LVDT was not used for the remainder of the experiments. The root mean square deviation data is presented in Table 1 .

Table 1: Root mean square deviation of LVDT and DIC displacement data

\begin{tabular}{|c|c|c|c|c|c|}
\hline Test & 1 & 2 & 3 & 4 & 5 \\
\hline RMSD [in] & 0.0005 & 0.0005 & 0.0006 & 0.0002 & 0.0004 \\
\hline
\end{tabular}

After the experiments were completed and the images were processed in Aramis software, it was observed that the strain distribution was significantly different from case to case as can be observed in Figure 4 . In order to investigate this difference in strain, two aluminum flat bars were examined, one with no perforation and one with the largest perforation. As discussed earlier, the GOM Aramis software specifies facets on the surface of the specimen and calculates strains using the displacement values of the facet centers. The method that is used in this paper extracts the facet data from Aramis software and considers them as the input data for calculating strains via least square technique, but first these displacement data has to be mapped onto a finite element mesh.

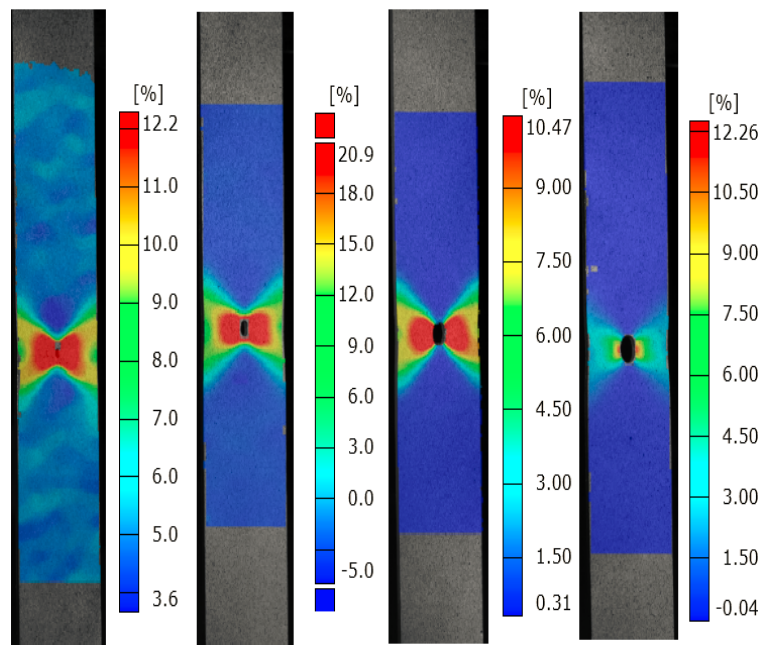

Figure 4: Longitudinal strain distribution on the aluminum specimen 


\section{Finite Element Approximation}

The facet data which are the displacement data obtained by DIC are extracted from the Aramis software post processing. These data are imported into MATLAB. A finite element mesh is generated on the imported facet data for both the initial and final configurations. The mesh for the final configuration of the displacement data is presented in Figure 5 .



Figure 5: Finite element mesh on the facet data

After the mesh was generated on the imported data, a least square method was used to find the best nodal displacement of the element. In order to be able to perform the least square approximation, all the elements have to include at least $n+1$ facets, where $n$ represents the number of element nodes. This procedure is briefly described here. The goal is to minimize the squared displacement differences as described in the following equation

$$
F=\sum_{i=1}^{N r E l m} \sum_{j=1}^{N F P}\left(\Delta \bar{u}^{*}-\Delta u^{D I C}\right)_{j}^{2},
$$

where NFP stands for number of facet points, $\Delta \bar{u}^{*}$ is the smoothed displacement over an element and $\Delta u^{D I C}$ is the vector of facet data inside each element. For each element $\Delta \overline{\boldsymbol{u}}^{*}$ is expressed as

$$
\Delta \bar{u}_{j}^{*}=N_{j i} \Delta u_{i}^{*} \quad \text { or } \quad \Delta \overline{\boldsymbol{u}}^{*}=\boldsymbol{N} \cdot \Delta \boldsymbol{u}^{*},
$$

where $\Delta \overline{\boldsymbol{u}}^{*}$ is the vector of nodal displacement values which is unknown at this stage. The minimum error is achieved when the derivative of the error function $F$ is set to zero,

$$
\begin{aligned}
\frac{\partial F}{\partial \Delta \boldsymbol{u}^{*}}=0 & \Longrightarrow \\
2\left(\Delta \bar{u}_{j}^{*}-\Delta u_{j}^{D I C}\right) \frac{\partial \Delta \bar{u}_{j}^{*}}{\partial \Delta u_{k}^{*}}=0 & \Longrightarrow \\
\left(N_{j i} \Delta u_{i}^{*}-\Delta u_{j}^{D I C}\right) N_{j k} & =0 .
\end{aligned}
$$

In this equation $N$ is the matrix of shape functions associated with the element and number of facet data inside the element. Finally this equation is expressed in terms of nodal displacements as

$$
\Delta \boldsymbol{u}^{*}=(\boldsymbol{N} \cdot \boldsymbol{N})^{-1} \cdot\left(\boldsymbol{N} \cdot \Delta \boldsymbol{u}^{D I C}\right)
$$

In order to solve this equation and find the best nodal displacement values, a search algorithm is used to locate the facet data associated with each element. To find the error of this method and find out what types of element could best represent the facet data, three types of elements were chosen: a four node quadrilateral element $\left(Q_{4}\right)$, an eight node serendipity element $\left(Q_{8}\right)$ and a nine node Lagrange element $\left(Q_{9}\right)$. The shape functions $(\boldsymbol{N})$ are defined as $\boldsymbol{P} \cdot \boldsymbol{C}^{-1}$. As an example for a $Q_{9}$ element $\mathrm{C}$ and $\mathrm{P}$ are defined as

$$
\boldsymbol{C}=\left(\begin{array}{cccccc}
1 & x_{1} & y_{1} & x_{1} y_{1} & \ldots & x_{1}^{2} y_{1}^{2} \\
1 & x_{2} & y_{2} & x_{2} y_{2} & \ldots & x_{2}^{2} y_{2}^{2} \\
1 & x_{3} & y_{3} & x_{3} y_{3} & \ldots & x_{3}^{2} y_{3}^{2} \\
1 & x_{4} & y_{4} & x_{4} y_{4} & \ldots & x_{4}^{2} y_{4}^{2} \\
1 & x_{5} & y_{5} & x_{5} y_{5} & \ldots & x_{5}^{2} y_{5}^{2} \\
1 & x_{6} & y_{6} & x_{6} y_{6} & \ldots & x_{6}^{2} y_{6}^{2} \\
1 & x_{7} & y_{7} & x_{7} y_{7} & \ldots & x_{7}^{2} y_{7}^{2} \\
1 & x_{8} & y_{8} & x_{8} y_{8} & \ldots & x_{8}^{2} y_{8}^{2} \\
1 & x_{9} & y_{9} & x_{9} y_{9} & \ldots & x_{9}^{2} y_{9}^{2}
\end{array}\right), \boldsymbol{P}=\left(\begin{array}{c}
1 \\
x \\
y \\
x y \\
x^{2} \\
y^{2} \\
x y^{2} \\
x^{2} y \\
x^{2} y^{2}
\end{array}\right) .
$$

In above equations $x_{i}$ and $y_{i}$ are $x$ and $y$ coordinates of the nodes of the elements and $\mathrm{x}$ and $y$ define the spatial position of the facet data inside the element. Finally the multiplication of matrix $\boldsymbol{C}^{-T}$ and vector $\boldsymbol{P}$ provides the value of the shape functions at the facet point of interest. The nodal positions are depicted in Figure 
6. In order to find out which type of element has a better representation of the facet data with less error, an error analysis was performed for $Q_{4}, Q_{8}$ and $Q_{9}$ element types based on the data of the non-perforated aluminum sample at the initial and final stage of the experiment.
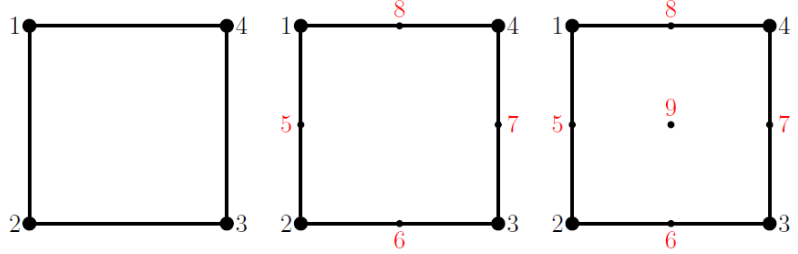

Figure 6: $Q_{4}, Q_{8}$ and $Q_{9}$ quadrilateral elements

The error analysis was performed for different number of elements starting from two and increasing to 128 elements as shown in Figure 7

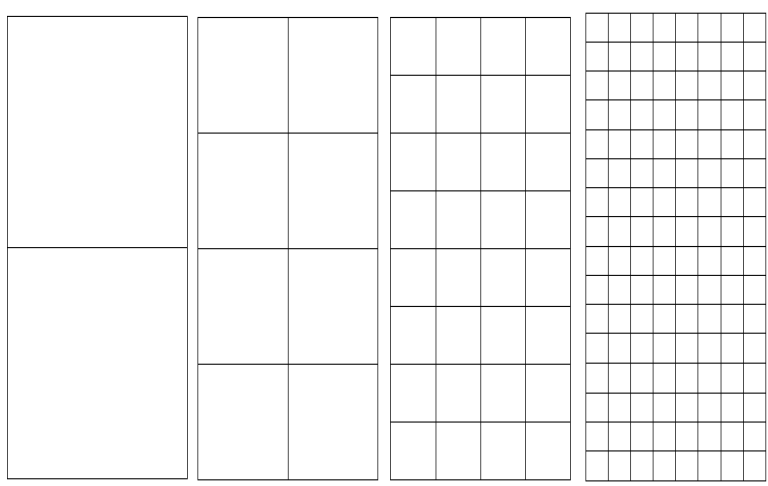

Figure 7: Finite element mesh with different number of elements

The results of error analysis for these three different element types is depicted in Figure 8. It should be noted that the horizontal axis of Figure 8 is a logarithmic axis in order to provide better presentation of the results. It is observed from this figure that error is decreasing by increasing the number of elements, however if the facet data inside the element are less than the number of nodes, the error is going to increase, i.e., increasing number of elements will only help if the number of facet data is sufficient for each element. It is also observed that the error associated with the $Q_{9}$ element is lower than the two other element types and therefore it is a better choice, however if number of elements is sufficiently large, the difference is minimal.



Figure 8: Error analysis based on least square for bilinear and biquadratic elements

Having the best nodal displacement values based on the DIC facet data, it is possible to calculate infinitesimal and finite strain distributions. In order to calculate small strain the gradient of the shape functions are calculated in each element and then multiplied by the nodal displacements as

$$
\boldsymbol{\epsilon}_{\text {ele }}=\boldsymbol{B} \cdot \Delta \boldsymbol{u}_{\text {ele }},
$$

where

$$
\boldsymbol{B}=\left(\begin{array}{ccccccc}
\frac{\partial N_{1}}{\partial x} & 0 & \frac{\partial N_{2}}{\partial x} & 0 & \ldots & \frac{\partial N_{i}}{\partial x} & 0 \\
0 & \frac{\partial N_{1}}{\partial y} & 0 & \frac{\partial N_{2}}{\partial y} & \ldots & 0 & \frac{\partial N_{i}}{\partial y} \\
\frac{\partial N_{1}}{\partial y} & \frac{\partial N_{1}}{\partial x} & \frac{\partial N_{2}}{\partial y} & \frac{\partial N_{2}}{\partial x} & \ldots & \frac{\partial N_{i}}{\partial y} & \frac{\partial N_{i}}{\partial x}
\end{array}\right)
$$

and

$$
\Delta \boldsymbol{u}_{\text {ele }}=\left(\begin{array}{lllll}
\Delta u_{x 1} & \Delta u_{y 1} & \ldots & \Delta u_{x i} & \Delta u_{y i}
\end{array}\right)^{T} .
$$

In order to calculate the finite strains two stages of the experiment are required, one defines the initial configuration and the other one the current configuration. Having the nodal coordinates of initial and current stages, the deformation gradients of displacements are calculated 
as

$$
\begin{array}{r}
\boldsymbol{F}(1: 2,1: 2)=\left(\begin{array}{l}
x_{1} \\
y_{1}
\end{array}\right)\left(\begin{array}{ll}
\frac{\partial N_{1}}{\partial x} & \frac{\partial N_{1}}{\partial y}
\end{array}\right)+ \\
\left(\begin{array}{l}
x_{2} \\
y_{2}
\end{array}\right)\left(\begin{array}{ll}
\frac{\partial N_{2}}{\partial x} & \frac{\partial N_{2}}{\partial y}
\end{array}\right)+\ldots+\left(\begin{array}{l}
x_{i} \\
y_{i}
\end{array}\right)\left(\begin{array}{ll}
\frac{\partial N_{i}}{\partial x} & \frac{\partial N_{i}}{\partial y}
\end{array}\right)
\end{array}
$$

and $\boldsymbol{F}(3,1: 2)$ and $\boldsymbol{F}(1: 2,3)$ are zero and $\boldsymbol{F}(3,3)$ is equal to one. Therefore the Green's (Lagrangian) finite strain tensor is expressed as

$$
\boldsymbol{E}^{G}=\frac{1}{2}(\boldsymbol{F} \cdot \boldsymbol{F}-\mathbf{1}) .
$$

Having the proper formulation, the facet data of an aluminum flat bar with circular perforation of $1 / 4$ of inch is extracted in order to perform the least square analysis. The finite element mesh and facet distribution in the vicinity of the perforation for the finite element mesh are depicted in Figures 9 and 10.
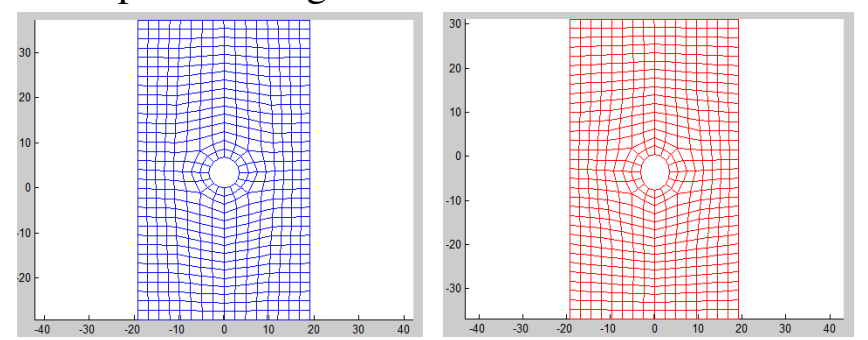

Figure 9: Initial and final mesh to cover the facet data in

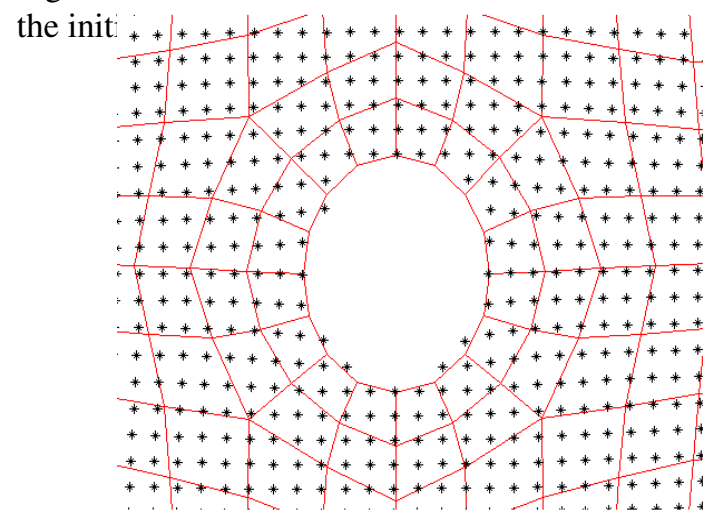

Figure 10: Facet distribution in the vicinity of perforation at the final stage

The comparison of the displacement distribution obtained by DIC and least square analysis is presented in Figure 11, the top two figures show the value of axial displacement of the DIC and LS approximation and the bottom two figures present the transverse displacement distribution.
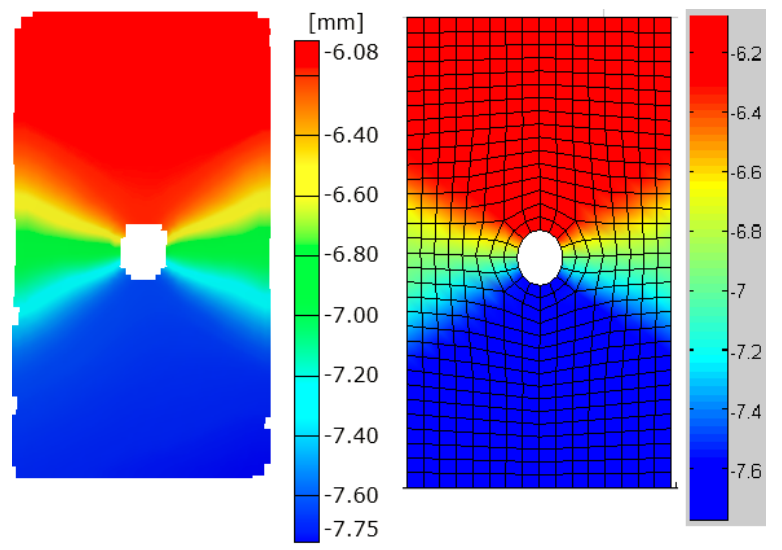

(a) Axial displacement

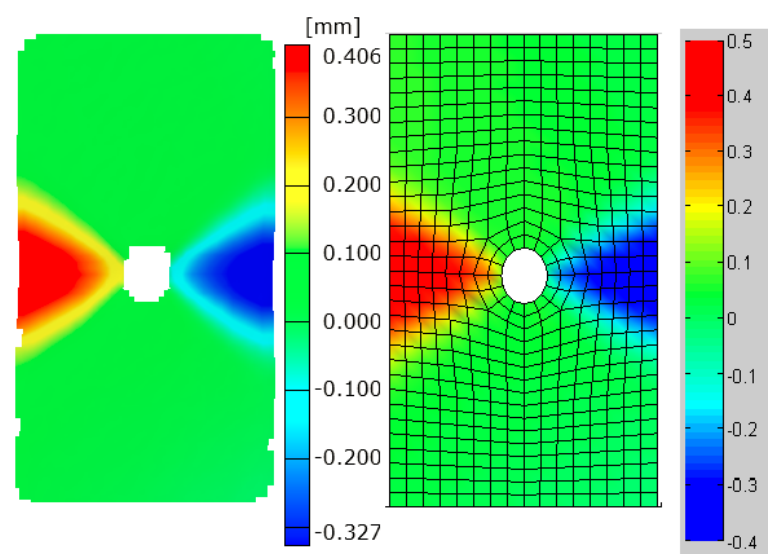

(b) Transverse displacement

Figure 11: Axial and transverse displacement distribution based on DIC and least square analysis

For this comparison, 128 elements and Q4 element type were used. It is observed that the least square approximation of the DIC facet data reproduces the displacement field with very small errors according to Figure 8 .

In order to compare the calculated strain of the Aramis software with the one predicted by the proposed method, two different facet sizes and distributions are used. These facets are selected to represent a fine and coarse displacement data over the experimental sample. The finite element mesh is kept the same for both of the cases. Finite strain distributions for the fine and coarse facet data were calculated by Aramis software is presented in Figure 12. It is observed that the finite strain distribution for the same experiment in the vicinity of the perforation is significantly different for the fine and 
coarse facet distribution.
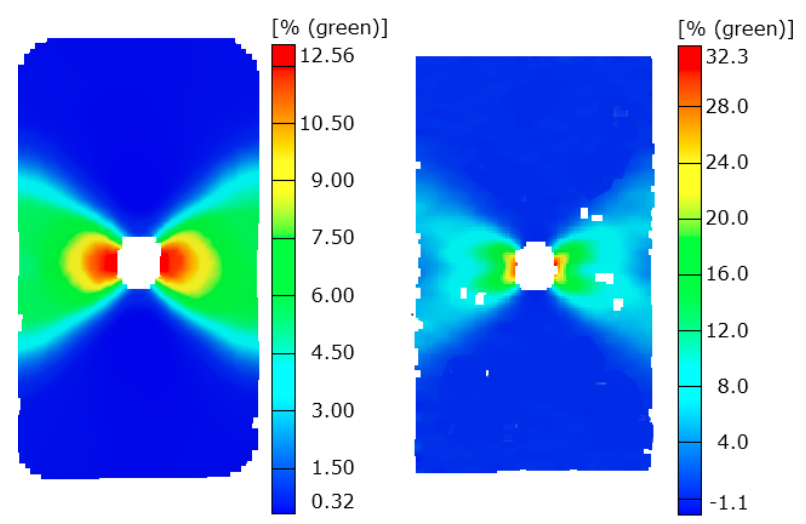

Figure 12: Axial large strain distribution, coarse and fine facet data in the left and right

The strain values in the fine case are 2.6 times greater than the strains in the coarse case. Now in order to check how these facet data are predicted by the proposed method in this report, least square analysis is performed for both cases. The result of this analysis is presented in Figure 13. As it can be seen, the predicted strain distribution for both of the input data is very similar. This shows that as long as there are enough facet data to perform a least square analysis and find the nodal displacements, the finite element approximation can calculate the strains with no sensitivity to facet density.
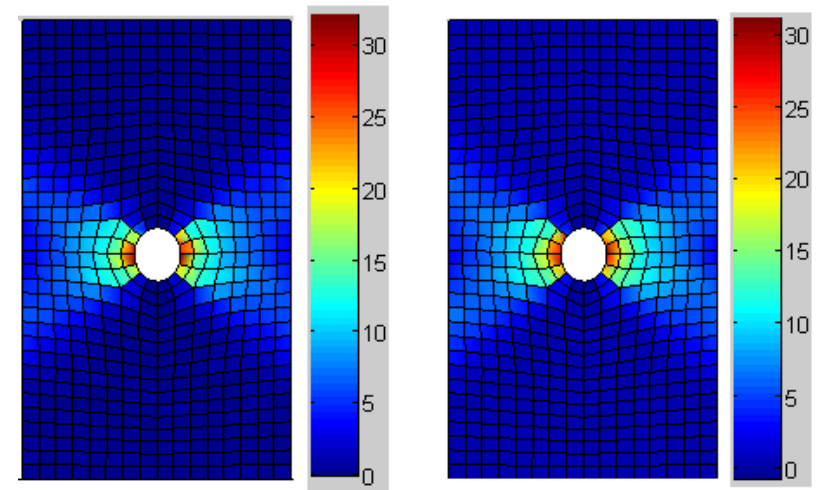

Figure 13: Axial large strain distribution calculated by finite element and least square analysis

\section{Concluding Remarks}

It was observed that the displacement data obtained by DIC system were similar to the results obtained by LVDT, as presented in Figure
3 and Table 11. The least square approximation of the finite element discretization covering the facet data for different element types, it was observed that the error associated with this method decreases while increasing the element numbers. however this was only true as long as there were enough facet data inside the element.

It was also observed that the error associated with Q9 elements was smaller than Q8 and $\mathrm{Q} 4$, but as the number of elements increases the difference in the errors were minimal as shown in Figure 8. The results of the finite and infinitesimal strain obtained by least square analysis were compared to the ones calculated by Aramis software. It was observed that although the displacements were in good agreement for both methods, the strains were significantly different. Therefore a study was conducted to see the effect of facet size and facet number on the strain distribution for these methods. It was observed that the strains obtained by post processing procedure of Aramis software is very sensitive to facet density and size, while the least square approximation provided very close results, i.e. insensitive results. The least square approximation of the displacement data provided quantitative results that are reflecting boundary and edge conditions much better than the native Aramis software.

The future work on this method is focused on comparing different stages of the experiment and calculating the strain distributions based on an updated Lagrange approach and to come up with appropriate methodologies to find localized failures on the specimen. This could be achieved in several ways, one would be to calculate the vorticity, shear and divergence distribution on the surface of the specimen as discussed by Peterson and Sulsky [21] and find the onset of discrete failure, in another way it is possible to calculate the strain rate from one stage to another stage and find the rank of the strain tensor by an eigenvalue analysis, if there exists a rank deficiency in the strain tensor, this could indicate a jump in the strain rate and therefore a sign of the loss of ellipticity in the material at that point. 


\section{REFERENCES}

[1] Chu, T. C., Ranson, W. F., Sutton, M. A. 1985. Applications of Digital-ImageCorrelation Techniques to Experimental Mechanics. Journal of Experimental Mechanics; 25 pp. 232-244.

[2] Lava, P., Cooreman, S., Coppieters, S., De Strycker, M., Debruyne, D. 2009. Assessment of Measuring Errors in DIC Using Deformation Fields Generated by Plastic FEA. Journal of Optics and Lasers in Engineering; 47 pp. 747-753.

[3] Yang, G., Zomorodian, M., Belarbi, A., Ayoub, A. S. 2015. Uniaxial Tensile Stress-Strain Relationships of RC Elements Strengthened with FRP Sheets. Journal of Composites for Construction.

[4] Corr, D., Accardib, M., Graham-Bradyc, L., Shah, S. 2007. Digital Image Correlation Analysis of Interfacial Debonding Properties and Fracture Behavior in Concrete. Journal of Engineering Fracture Mechanics; 74 pp. 109-121.

[5] Pan, B., Qian, K., Xie, H., Asundi, A. 2009. Two-dimensional Digital Image Correlation for In-plane Displacement and Strain Measurement:a Review. Measurement Science and Technology.

[6] Ghiassi, B., Xavier, J., Oliveira, D. V., Lourenoa, P. B. 2013. Application of Digital Image Correlation in Investigating the Bond Between FRP and Masonry. Journal of Composite Structures; 106 pp. 340-349.

[7] Champiri, M. D., Mousavizadegan, S. H., Moodi, F. 2012. A Decision Support System for Diagnosis of Distress Cause and Repair in Marine Concrete Structures. International Journal of Computers and Concrete; pp. 99-118.

[8] Champiri, M. D., Mousavizadegan, S. H., Moodi, F. 2012. A Fuzzy Classification System for Evaluating Health Condition of Marine Concrete Structures. Journal of Advanced Concrete Technology; 10 pp. 95-109.

[9] Roncella, R., Romeo, E., Barazzetti, L., Gianinetto, M., Scaioni, M. 2012. Comparative Analysis of Digital Image Correlation Techniques for In-plane Displacement Measurements. 5th International Congress on Image and Signal Processing; pp. 721-726.

[10] Beizaee, S., Xotta, G., Willam, K. J. 2016. Perforation Studies on Flat Bars for XFEM-Based Failure Analysis. Journal of Engineering Fracture Mechanics; 155 pp. 67-87.

[11] Xotta, G., Beizaee, S., Willam, K. J. 2016. Bifurcation Investigations of Coupled Damage-Plasticity Models for Concrete Materials. Computer Methods in Applied Mechanics and Engineering; 298 pp. 428-452.

[12] Beizaee, S., Willam, K. J., Xotta, G. 2013. The Effect of Circular Openings on the Brittle-Ductile Fracture of Ferrous Flat Bars. VIII International Conference on Fracture Mechanics of Concrete and Concrete Structures FraMCoS-8.

[13] Karagah, H., Shi, C., Dawood, M., Belarbi, A. 2015. Experimental Investigation of Short Steel Columns with Localized Corrosion. Journal of Thin-Walled Structures; 87 pp. 191-199.

[14] Shi, C., Karagah, H., Dawood, M., Belarbi, A. 2014. Numerical Investigation of H-shaped Short Steel Piles with Localized Severe Corrosion. Journal of Engineering Structures; 73 pp. 114-124.

[15] Mohammadipour, A. H., Willam, K. J., Ayoub, A.S. 2013. Experimental Studies of Brick and Mortar Composites Using Digital Image Analysis. Processing of $8^{\text {th }}$ 
International Conference of Fracture Mechanics of Concrete and Concrete Structures; pp. 183-192.

[16] Willam, K. J., Mohammadipour, A., Mousavi, R., Ayoub, A.S. 2013. Failure of Unreinforced Masonary Under Compression. Structures Congress, ASCE; pp. 2949-2961.

[17] ASTM B557-15, Standard Test Methods for Tension Testing Wrought and Cast Aluminum- and Magnesium-Alloy Products, ASTM International, West Conshohocken, PA, 2015, www.astm.org

[18] ASTM A370-15, Standard Test Methods and Definitions for Mechanical Testing of
Steel Products, ASTM International, West Conshohocken, PA, 2015, www.astm.org

[19] ASTM E8 / E8M-15a, Standard Test Methods for Tension Testing of Metallic Materials, ASTM International, West Conshohocken, PA, 2015, www.astm.org

[20] Aramis Software Manual, GOM Optical Measuring Techniques Company.

[21] Peterson, K. and Sulsky, D. 2011. Evaluating Sea Ice Deformation in the Beaufort Sea Using a Kinematic Crack Algorithm with RGPS Data. Remote Sensing of the Changing Oceans; pp. 123139. 